\title{
Effects of Size of Interlacer on Air Flow in a Yarn Duct
}

\author{
IEMOTO Yoshiyuki*, TANOUE Shuichi, Lu Jun, TAKANO Tetsuji \\ Graduate School of Engineering, University of Fukui, 3-9-1 Bonkyo, Fukui-shi 910-8507, Japan
}

Received 27 December 2008; accepted for publication 12 May 2009

\begin{abstract}
In order to make clear the air flow in the yarn duct, which has the maximum effect on the generation of interlaced yarns, the flow near to the yarn duct wall and the jet issuing from a circular air jet nozzle were measured in basic type interlacers with various diameters and lengths of yarn duct. The jet issuing from the air jet nozzle forms a detached shock wave in front of the yarn duct wall opposite to the air jet nozzle. After running against the yarn duct wall, the air forms two twin spiral eddies in the yarn duct. The diameter ratio of yarn duct to air jet nozzle has an effect on the jet width and the circumferential component of the flow velocity and hence it has a greater effect on the processability of interlaced yarns than the yarn duct length.
\end{abstract}

Key Words: Interlacer, High speed air flow, Two twin spiral eddies, Detached shock wave

\section{Introduction}

Interlacers are composed of three parts: a yarn duct, an air jet nozzle and yarn guides as shown in Fig. 1. A high-speed air jet from the air jet nozzle hits against a multi-filament yarn running at a constant speed in the yarn duct and then makes an interlaced yarn with tangling and opening parts shown in Fig. 2. Since the interlaced yarn is cohesive, fabrics are woven or knitted from it without sizing or twisting. Moreover, it is used as a yarn material of specialty fabrics because the interlacing process can combine two or more yarns with different properties and functions.

Interlacers with a variety of shapes and sizes have been developed and put to practical use because a long time has passed after the interlacing process was developed in $\mathrm{Du}$ Pont [1]. However, the processing mechanism is still unclear

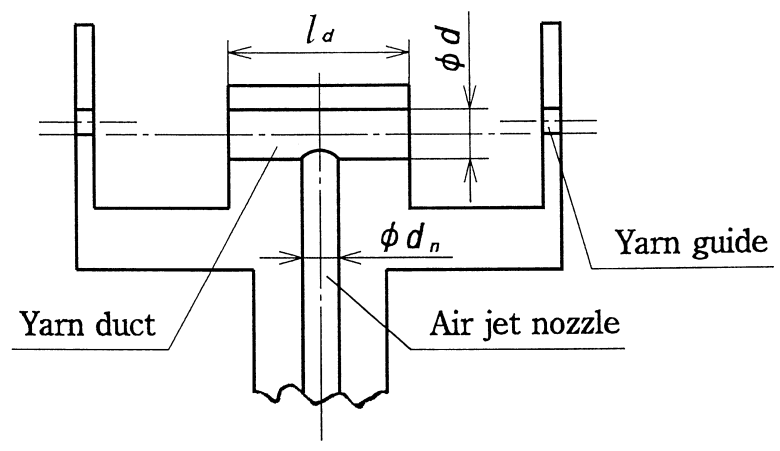

Fig. 1 Basic type interlacer.

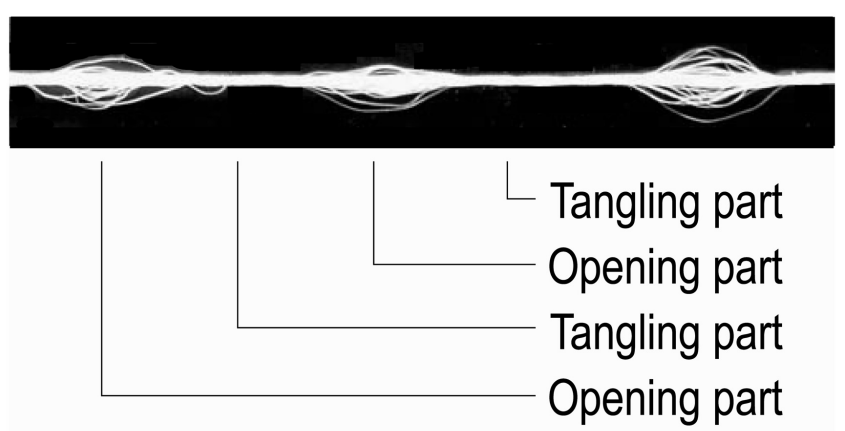

Fig. 2 Interlaced yarn.

and the optimum interlacer has not been obtained yet.

In order to clarify the processing mechanism, we have carried out experimental analyses of a yarn motion in basic type interlacers, where a circular air jet nozzle is installed at the center of a circular yarn duct [2,3], model experiments [4-8], and discussion of structure and properties of interlaced yarns [9-14]. However, since the interlaced yarn is generated by air force, it is indispensable to clarify air flow. Lünenschloß and Zilg [15] measured the distribution of air static pressure on the yarn duct wall using an interlacer with practical size, although the measurement is difficult because the size of yarn duct is small, the air flow velocity is extremely high and the flow is three-dimensional. We also measured the distributions of static pressure on the yarn duct wall of a one-sized interlacer and those of dynamic pressure and flow direction near to the same wall [16]. Moreover, we examined the static pressure distribution on the yarn duct

\footnotetext{
* Corresponding author: E-mail: iemoto@matse.u-fukui.ac.jp, Fax: +81-776-27-8767
} 
wall of interlacers with various sizes [17]. Murakami et al. [18] measured the distributions of static pressure on the yarn duct wall opposite to the air jet nozzle and velocity near to the same wall and numerically simulated incompressible air flow in the case of very small supplied air pressure and flow rate, and furthermore Tokunaga et al. [19] measured the static pressure distribution on the yarn duct wall opposite to the air jet nozzle and numerically simulated subsonic flow in the case of relatively low supplied air pressure under 0.2 $\mathrm{MPa}$. Although such numerical simulations were carried out, flow measurement on the condition of the practical supplied air pressure is limited to the static pressure and a flow such as air dynamic pressure and flow direction in interlacers with various sizes has not been measured.

In this study, we clarified effects of sizes of yarn duct and air jet nozzle on the flow in the yarn duct using basic type interlacers. Here, the measurement was carried out without running a yarn because it was much smaller than the yarn duct diameter. We used similarly enlarged interlacers because practical interlacers are too small to measure pressures at high accuracy. First of all, the dynamic pressure and flow direction near to the yarn duct wall of interlacers with various diameters and lengths of yarn duct were measured with a Pitot tube. Next, the air flow at the section of air jet nozzle was measured with the Pitot tube and a Schlieren technique which has not been applied to the air flow measurement in interlacers. This is because the air flow at the section plays a more important role in the generation of interlaced yarn than at other sections: an air jet separates a yarn and then makes an opening part, furthermore tangling parts are formed on both sides of the opening part near the section of air jet nozzle [2].

\section{Experimental apparatus and procedure}

The route of a compressed air is shown in Fig. 3. The air compressed by a compressor (1) passed through a valve (2), was adjusted to an arbitrary pressure with a pressure

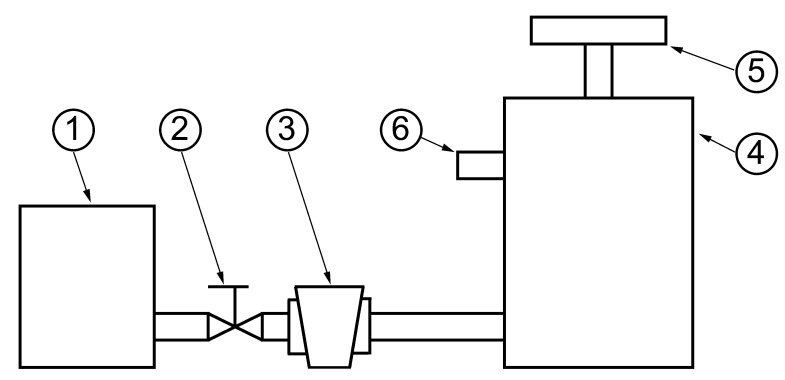
(1) Compressor
(2) Valve
(3) Pressure regulator
(4) Rectification tank
(5) Interlacer
(6) Pressure transducer

Fig. 3 Route of compressed air.
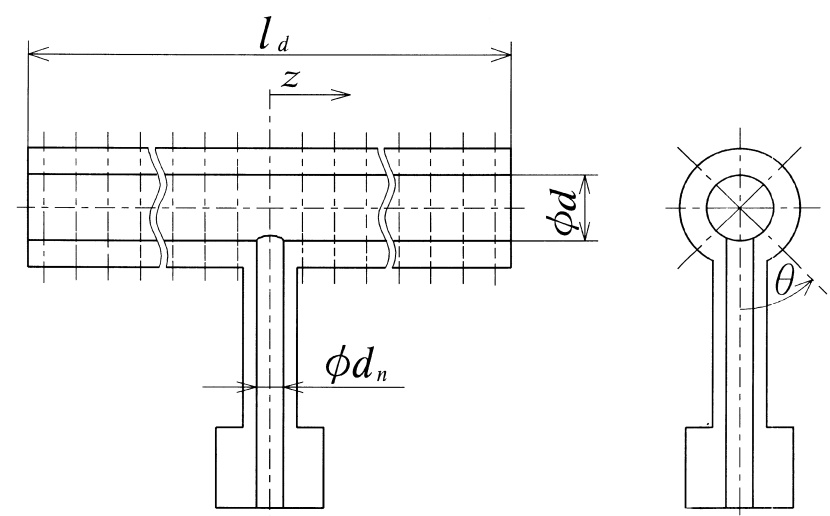

Fig. 4 Measurement points in enlarged interlacer and coordinate system.

regulator (3), and arrived at a rectification tank (4). An interlacer (5) was installed at the top of the rectification tank, and the air flowed out to the atmosphere through an air jet nozzle and a yarn duct. A strain-gauge type pressure transducer (6) was used for the measurement of the supplied air pressure in the rectification tank.

Since practical interlacers are too small to measure the flow in the yarn duct at high accuracy, similarly enlarged interlacers were used as in the previous papers [16, 17]. The basic type interlacers shown in Fig. 4 were made of acrylic resin. The diameter $d_{n}$ of the air jet nozzle was fixed to $7.3 \mathrm{~mm}$. The diameter ratio $R_{d}\left(=d / d_{n}\right)$ of the yarn duct diameter $d$ to $d_{n}$ and the non-dimensional yarn duct length $L_{d}\left(=l_{d} / d\right)$ were changed as follows: $R_{d}=1.0,1.3,1.6,2.0,2.4$ and $L_{d}=3.7$, $5.0,7.0,11.0,15.0$. The law of similarity of the air flow in practical interlacers and enlarged ones is effective because the enlargement ratios of the interlacers were several times [17].

In order to measure the pressure on the yarn duct wall and the total pressure near to the same wall, small holes of $0.8 \mathrm{~mm}$ in diameter were made in the yarn duct wall at intervals of $d / 2$ in the direction of the yarn duct axis and $45^{\circ}$ in the circumferential direction. The coordinate axes $\theta$ and $z$ are defined to represent measurement positions shown in Fig. 4. The small holes were directly used to measure the wall pressure. A cylindrical Pitot tube of $0.8 \mathrm{~mm}$ in diameter was used to measure the total pressure as shown in Fig. 5. The Pitot tube, in which a pressure hole of $0.2 \mathrm{~mm}$ in diameter was made at the position $1 \mathrm{~mm}$ apart from the tip of the tube, was inserted to locate the pressure hole at the position $1 \mathrm{~mm}$ apart from the yarn duct wall. Pressure readings varied when the Pitot tube was rotated about its own axis. The maximum pressure, which was defined as a total pressure, and the rotational angle $\theta_{p}$, which shows a flow direction, were measured. The dynamic pressure was obtained by subtracting the wall pressure from the total pressure. The strain gauge type pressure transducer and the 


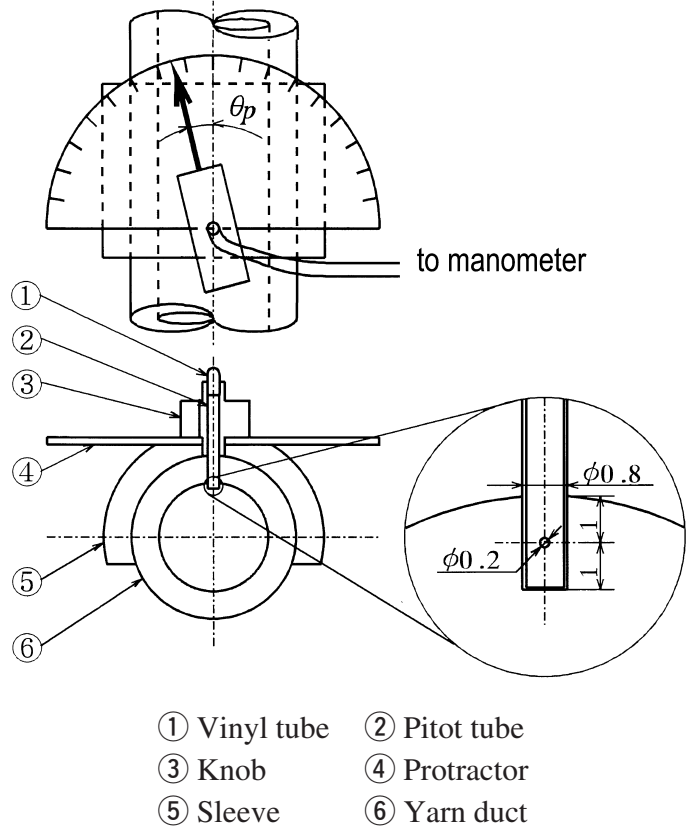

Fig. 5 Pitot tube.

mercury manometer were used to read the wall and total pressures.

After the flow in the yarn duct was confirmed to be symmetric with respect to both the plane of $z=0$ and the plane of $\theta=0$ and $180^{\circ}$, only a quarter region on the yarn duct wall was measured. In order to make clear effects of yarn duct length on the flow, we repeated to cut the yarn duct short and to measure the flow after we began the measurement from the interlacer with the longest yarn duct.

In the experiment the supplied air pressures $p$ was changed as follows: $p=0.2,0.3,0.4,0.5,0.6 \mathrm{MPa}$ in gauge pressure.

The measurement was carried out without running a yarn as in the previous papers [15-19] because the yarn was much smaller than the yarn duct diameter and the existence of the yarn has hardly any effect on the flow.

Moreover, to make clear the width of jet issuing from the air jet nozzle into the yarn duct, the position where the flow direction changes was measured by inserting the abovementioned Pitot tube through the small hole located at $\theta=$ 90 or $270^{\circ}$ of the section $z=0$ into various positions in the yarn duct while the pressure hole of the Pitot tube faces the air jet nozzle or its opposite. The jet width at the section $z=$ 0 was measured by this method. The yarn duct length used for the measurement is $L_{d}=5.0$.

In addition, the gradient of air density at the section $z=0$ in the yarn duct was observed by throwing light in the axial direction of the yarn duct by means of a Schlieren technique. The yarn duct length used was the shortest one of $L_{d}=3.7$ because the long yarn ducts blocked off the light.

\section{Results and discussion}

\section{1 Dynamic pressure and direction of flow near to yarn duct wall}

Figure 6 shows examples of effects of the diameter ratio $R_{d}$ on the dynamic pressure and direction of the flow near to the yarn duct wall. Figure 6(a) is a result for the nondimensional yarn duct length $L_{d}=5.0$ and the supplied air pressure $p=0.4 \mathrm{MPa}$, and Fig. 6(b) is for $L_{d}=7.0$ and $p=$ $0.2 \mathrm{MPa}$. These figures show a quarter of the yarn duct wall which is cut along $\theta=0$ and $180^{\circ}$ and made flat. The abscissa is the non-dimensional axial coordinate $Z(=z / d)$ and the ordinate is the angle $\theta$ in the circumferential direction shown in Fig. 4. The length scale of the ordinate is the same as that of the abscissa. That is, the length from $0^{\circ}$ to $180^{\circ}$ of the ordinate is $\pi / 2$ times the unit length of the abscissa. The black parts at $Z=0$ and $\theta=0^{\circ}$ in the figures show the exit of air jet nozzle. The direction and length of an arrow in the figure show a flow direction and a dynamic pressure corresponding to a flow velocity, respectively.

The compressed air jetting from the air jet nozzle into the yarn duct runs vertically against the duct wall opposite to the air jet nozzle and spreads along the yarn duct wall and radially from the central point of $Z=0$ and $\theta=180^{\circ}$. This flow phenomena are independent of the values of $R_{d}, L_{d}$ and $p$. In particular, even the case that the yarn duct diameter is the same as the air jet nozzle diameter $\left(R_{d}=1\right)$ is not exception. The air with a circumferential velocity component flows along the curved wall and collides with each other at the position $\theta=0^{\circ}$ because the yarn duct is cylindrical. Afterward, it flows by way of the center of the yarn duct to the position $\theta=180^{\circ}$ and again turns to $\theta=90$ and $270^{\circ}$. That is, two twin eddies are formed in the yarn duct, the air flows spirally and then out of the yarn duct edge to the atmosphere.

The flow velocity is zero at the wall center opposite to the air jet nozzle $\left(Z=0\right.$ and $\left.\theta=180^{\circ}\right)$ and increases with the distance from the wall center. The position of the maximum flow velocity on the line $\theta=180^{\circ}$ is in $Z=0.5-1.5$ although this value is not strict because the intervals of measurement points are large. After that position, the velocity monotonically decreases with an increment in the distance from the wall center. The maximum of the velocity also occurs in the circumferential direction and as a result, the position of the maximum flow velocity forms a circumference of circle whose center is at the wall center. The pressure on the wall becomes minimum on the circumference of the circle [17]. The axial flow velocity is large at $\theta=180^{\circ}$ in the region of small $|Z|$ excluding the neighborhood of the air jet nozzle exit $|Z| \leqq 1$ and the flow 


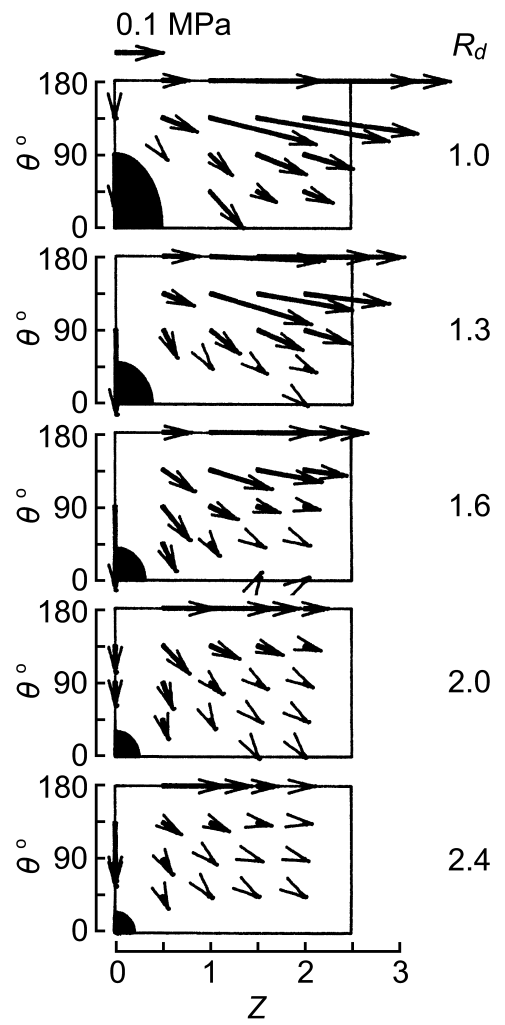

(a) Yarn duct length $L_{d}=5.0$ and supplied air pressure $p=0.4 \mathrm{MPa}$

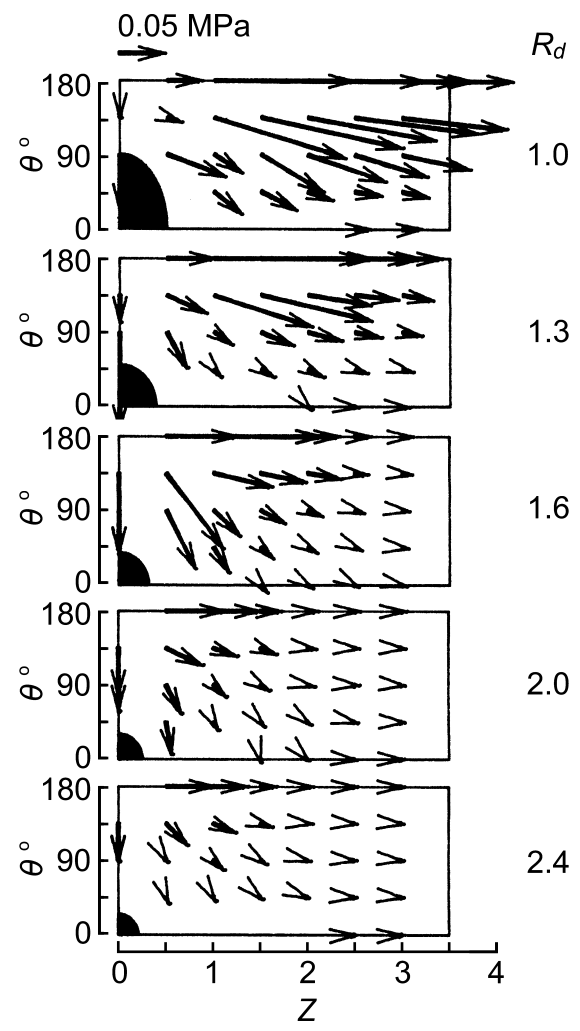

(b) Yarn duct length $L_{d}=7.0$ and supplied air pressure $p=0.2 \mathrm{MPa}$

Fig. 6 Effects of diameter ratio $R_{d}$ on the dynamic pressure and direction of flow near to yarn duct wall.

is biased toward $\theta=180^{\circ}$. However, as $|Z|$ is large, the circumferential component of the velocity becomes small and the axial component becomes predominant and uniform.

When $R_{d}$ is large, the dynamic pressure is small. When the supplied air pressure is constant, the air flow rate issuing from the air jet nozzle does not vary and hence the air flow rate through the yarn duct does not vary. When $R_{d}$ is large and $p$ is constant, the flow area is large and hence the flow velocity is small owing to the condition of continuity. The larger $R_{d}$ is, the more the air in the region of small $|Z|$ flows in the circumferential direction. This is because the flow space is large, the influence of the curved surface of the yarn duct is relatively small, and as a result, the circumferential component of velocity is comparable with the axial component of velocity.

Figure 7 shows effects of the supplied air pressure $p$ on the dynamic pressure and direction of flow near to the yarn duct wall. The interlacer with the diameter ratio $R_{d}=2.0$ and the non-dimensional yarn duct length $L_{d}=5.0$ was used in this example figure. Since the flow rate is large for large $p$, the position where the dynamic pressure is the maximum is far from the wall center and the flow velocity is large in $|Z|$ $\geqq 1$. However, the dynamic pressure in $|Z| \leqq 0.5$ is not monotonously changed with an increase in $p$ because expansion, compression and shock waves are generated in

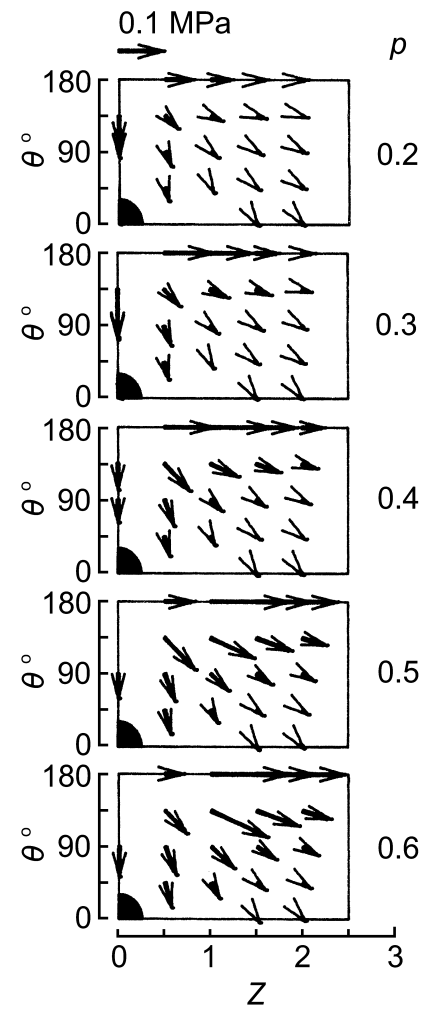

Fig. 7 Effects of supplied air pressure $p$ on the dynamic pressure and direction of flow near to yarn duct wall for diameter ratio $R_{d}=2.0$ and yarn duct length $L_{d}=5.0$. 


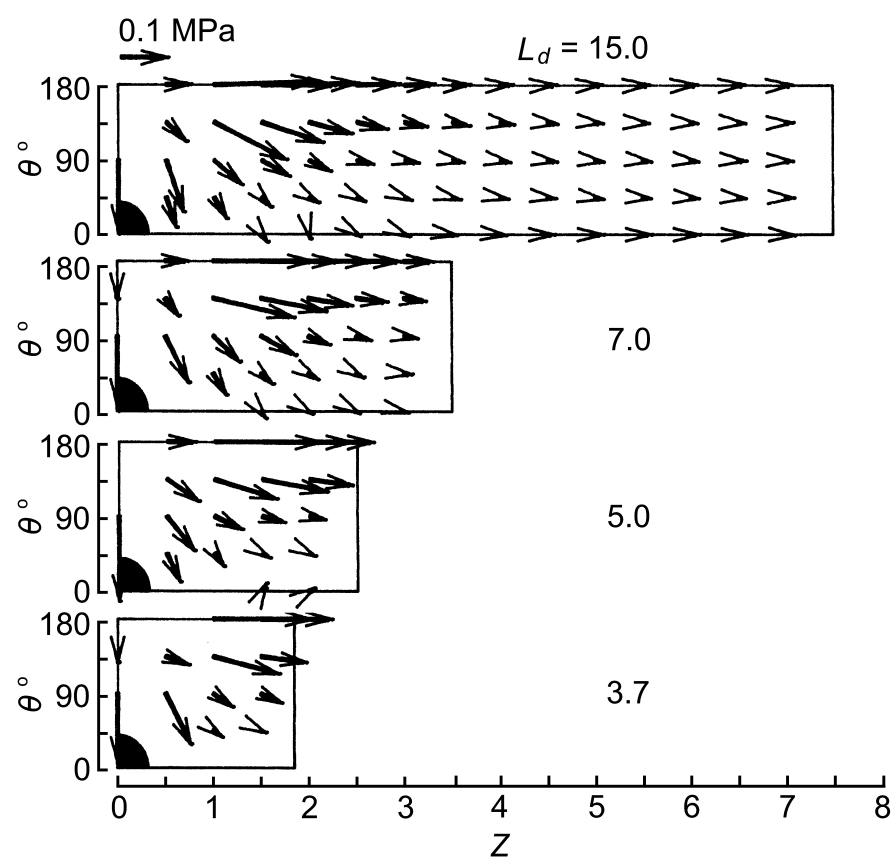

(a) Diameter ratio $R_{d}=1.6$ and supplied air pressure $p=0.4 \mathrm{MPa}$

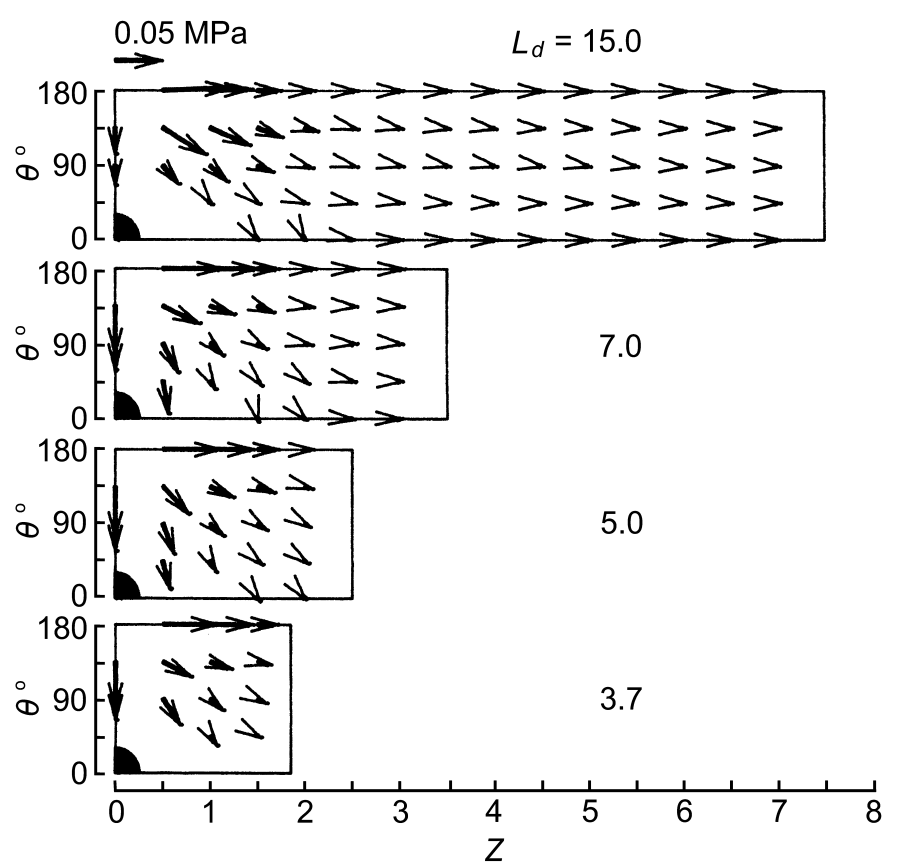

(b) Diameter ratio $R_{d}=2.0$ and supplied air pressure $p=0.2 \mathrm{MPa}$

Fig. 8 Effects of yarn duct length $L_{d}$ on the dynamic pressure and direction of flow near to yarn duct wall.

the flow from the air jet nozzle as shown in the following section. The flow direction is independent of $p$.

Figure 8 shows effects of the non-dimensional yarn duct length $L_{d}$ on the dynamic pressure and direction of flow near to the yarn duct wall. Figure $8(a)$ is a result for the diameter ratio $R_{d}=1.6$ and the supplied air pressure $p=0.4 \mathrm{MPa}$ and Fig. 8(b) is for $R_{d}=2.0$ and $p=0.2 \mathrm{MPa}$. When $L_{d}$ is as small as 3.7, the flow is biased to the line $\theta=180^{\circ}$, is not in the axial direction and shows a steep distribution even near to the yarn duct edge. The flow distribution near to the edge decreases with an increase in $L_{d}$. When $R_{d}$ is small and $p$ is large, the flow distribution near to the edge hardly disappears. However, when $L_{d}$ is as large as 15.0, the flow near to the edge is axisymmetric and is not biased, and the air flows out to the atmosphere in the axial direction even for $R_{d}=1.0$ and $p=0.6 \mathrm{MPa}$. The air at the same position $Z$ of interlacers with different $L_{d}$ flows at the same dynamic pressure in the same direction independently of $L_{d}$. That is, it can be suggested that the existence of the yarn duct edge has no effect on the flow in the yarn duct except for a small region near to the yarn duct edge.

\section{2 Jet width}

The most important position to make interlaced yarns is in front of the air jet nozzle in the yarn duct because opening parts of interlaced yarn are formed at the position where the air jet issuing from the air jet nozzle hits against a yarn, and tangling parts are formed on both sides of the opening part.

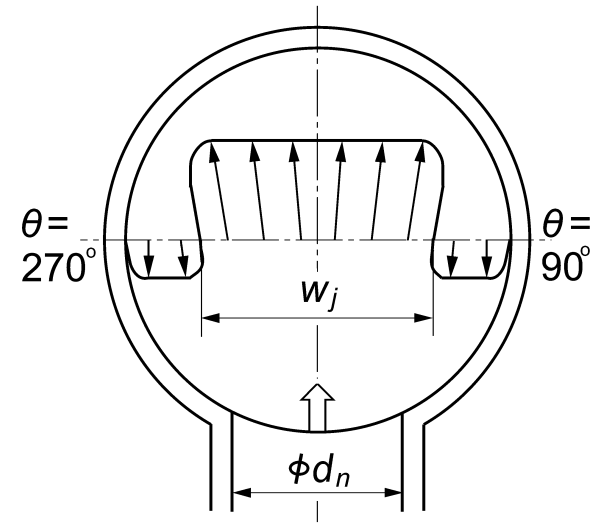

Fig. 9 Definition of jet width $w_{j}$ at the cross-section $Z=0$.

Therefore, we will discuss the jet width at the section $Z=0$ of the yarn duct where the air jet nozzle is installed. As shown in Fig. 9, the jet width $w_{j}$ defined in this section does not mean a core flow width, but means a width, in which the air flows from the air jet nozzle to its opposite wall, on a straight line through $\theta=90$ and $270^{\circ}$ at the section of $Z=0$, that is, the width including an accompanied flow.

Figure 10 shows the relation between the non-dimensional jet width $W_{j}$ and the diameter ratio $R_{d}$. The value of $W_{j}$ is the jet width divided by $d_{n}$. The non-dimensional yarn duct length is $L_{d}=5.0$ in the experiment. The value of $W_{j}$ increases in almost a straight line with $R_{d}$. When $R_{d}<1.5$, then $W_{j}<1$, the jet width shrinks from the air jet nozzle diameter, and the reverse flow regions occur near to the duct walls of $\theta=90$ and $270^{\circ}$. On the other hand, when $R_{d}>1.5$, the jet width expands from the air jet nozzle diameter. 


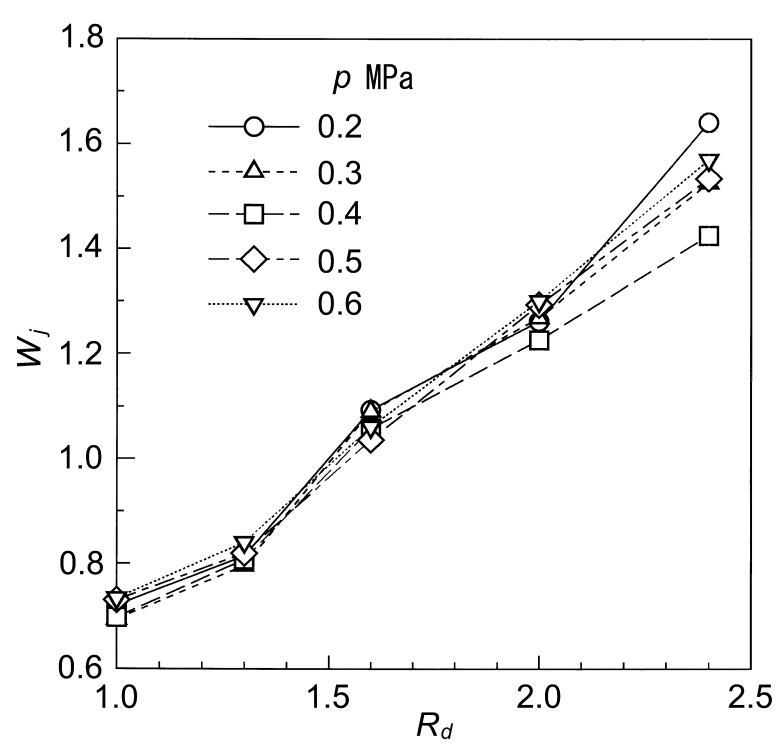

Fig. 10 Jet width $W_{j}$ as a function of $R_{d}$ for $L_{d}=5.0$.

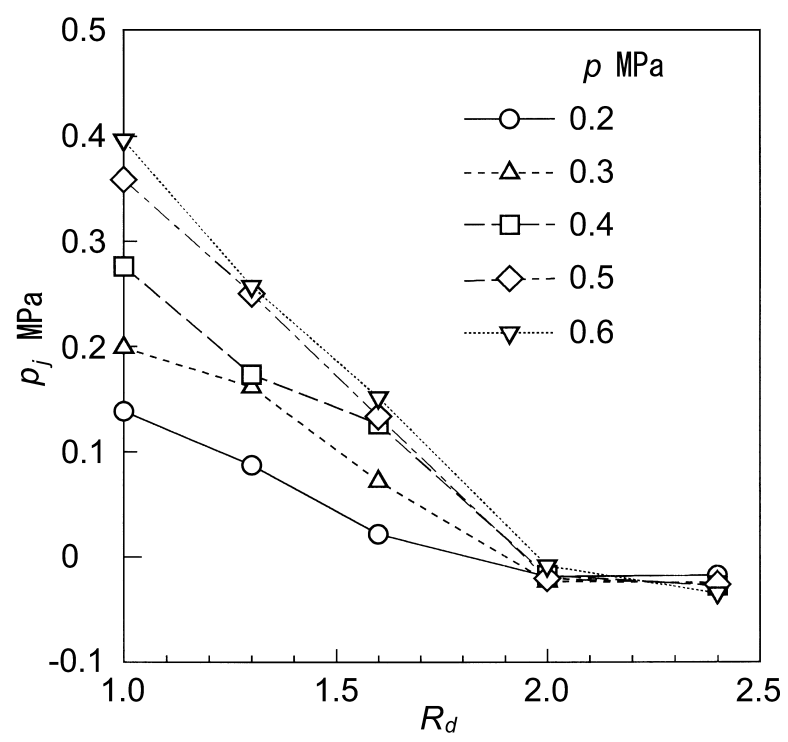

Fig. 11 Total pressure $p_{j}$ on jet boundary as a function of $R_{d}$ for $L_{d}=5.0$.

The size $R_{d}-W_{j}$ of reverse flow region also increases monotonously with $R_{d}$. It is can be said that the jet width is independent of $p$ although it varies slightly with $p$ in the interlacer with $R_{d}=2.4$.

Figure 11 shows the total pressure $p_{j}$ on the boundary of jet and reverse flow against the diameter ratio $R_{d} . p_{j}$ decreases with an increment in $R_{d}$. However, in the case that a large reverse flow region is generated $\left(R_{d}=2.0-2.4\right), p_{j}$ is nearly the same negative value. The air at the jet boundary takes negative pressure because it is accompanied by the expanded jet. The negative pressure was also observed on the yarn duct wall of $\theta=90$ and $270^{\circ}$ in the previous paper [17]. $p_{j}$ increases with $p$, but the difference of $p_{j}$ decreases with an increase in $R_{d}$ and almost vanishes when $R_{d}=2.0-2.4$.

Since the above-mentioned measurement was done by inserting the Pitot tube through the yarn duct wall into the yarn duct, the flow is disturbed by the Pitot tube. Moreover, since the shock wave occurs when the Pitot tube is inserted into a supersonic flow, it is necessary to correct the measurement values. However, it is difficult. Although the measurement values are not strictly correct, it seems that the outline of the flow is caught.

\section{3 Gradient of air density at the section of the air jet nozzle}

Figure 12 is examples of Schlieren photographs which show the gradient of air density at the section $Z=0$. The experimental conditions are $R_{d}=2.0$ and $p=0.3 \mathrm{MPa}$ in Fig. 12(a), $R_{d}=2.0$ and $p=0.6 \mathrm{MPa}$ in Fig. 12(b), and $R_{d}=$ 1.6 and $p=0.6 \mathrm{MPa}$ in Fig. 12(c). For small $p$ as shown in Fig. 12(a), the air issuing from the air jet nozzle located in the lower part of the figure forms a jet in the yarn duct and a detached shock wave in front of the duct wall opposite to the nozzle. The width of core flow is smaller than the diameter of air jet nozzle. As shown in Fig. 12(b), however, the width increases with $p$ and expands from the exit of air jet nozzle. For small $R_{d}$ as shown in Fig. 12(c), the boundary of core flow could not be photographed, but the formation of a detached shock wave was clearly observed.

Figure 13 shows the non-dimensional core flow width $C_{j}$ on the line of $\theta=90$ and $270^{\circ}$. The width $C_{j}$ is nondimensionalized by the diameter of air jet nozzle. Only

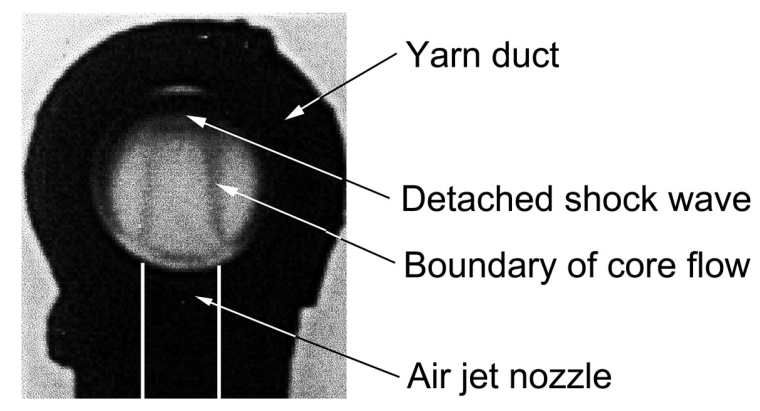

(a) $R_{d}=2.0$ and $p=0.3 \mathrm{MPa}$

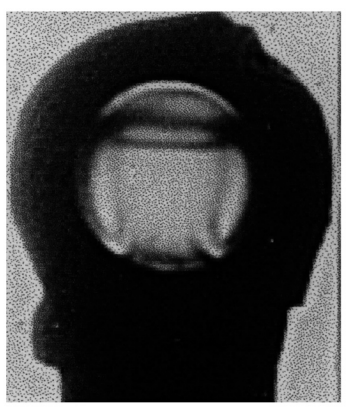

(b) $R_{d}=2.0$ and $p=0.6 \mathrm{MPa}$

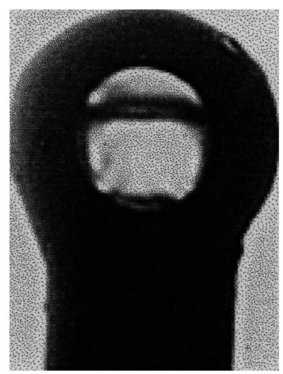

(c) $R_{d}=1.6$ and $p=0.6 \mathrm{MPa}$
Fig. 12 Schlieren photographs for $L_{d}=3.7$. 


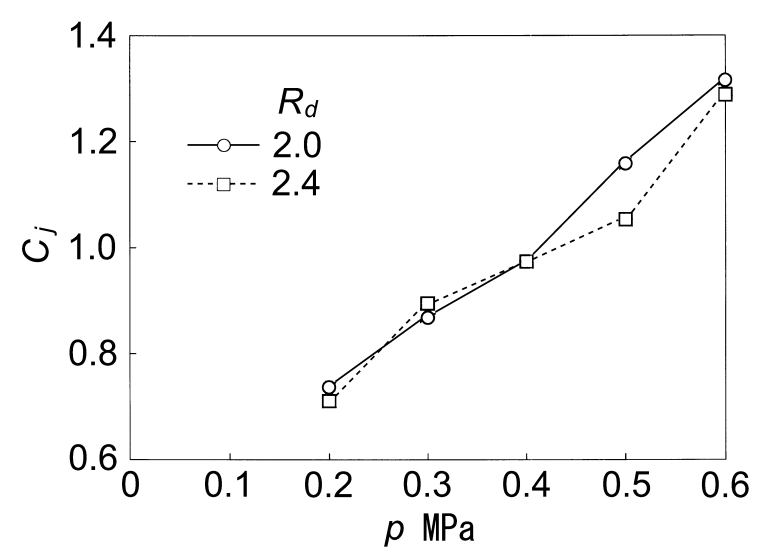

Fig. 13 Core flow width of jet $C_{j}$ for $L_{d}=3.7$.

results for $R_{d}=2.0$ and 2.4 were drawn in the figure. This is because the boundary of core flow could not be clearly observed as mentioned above when $R_{d}$ is smaller than those values. The width is smaller than the diameter of air jet nozzle for small $p$, but almost linearly increases with $p$ and swells from the exit of air jet nozzle for large $p$. In the range of this experiment the width is independent of $R_{d}$, but is supposed to be small for small $R_{d}$ because the space of the flow is narrow.

\section{4 Air flow and processability}

Processability of interlaced yarns will be discussed on the basis of the above-mentioned flow results.

When $p$ is large, the jet force acting on a yarn is large and the forces which act on filaments located at different positions are different because the spatial change in density of air jetting from the air jet nozzle is great. Hence, the opening and tangling action is large for large $p$. However, when the air jet force is too strong, already generated tangling parts are occasionally destroyed [8].

When $R_{d}$ is small, the flow velocity is large and hence the force acting on a yarn is large. However, when $R_{d}$ is too small, the air jet always hits against a yarn and already generated tangling parts are easily destroyed. $R_{d}$ needs to take the optimum value for the already generated tangling parts to pass through the section of the air jet nozzle without being exposed to the jet. When $R_{d}$ is large, the air force has no effective action on the yarn.

When a yarn is located on the side of $\theta=180^{\circ}$, the air frictional force acting on the yarn in the direction of the yarn axis is large because the axial flow velocity is large. When the yarn is located on the side of $\theta=0^{\circ}$, the air frictional force is small. The yarn moves in the modified form of letter 8 at the section of the air jet nozzle and in the spiral form in the direction of the yarn duct axis [2]. Hence, the axial tension of yarn varies depending on the yarn position. When
$L_{d}$ is large, the region where the air flow acts on the yarn is long and hence the air frictional force acting on the yarn in the direction of the yarn axis is large and weakens the opening action of air. As a result, it may be meaningless to lengthen $L_{d}$ more than a required length.

Since the most important position to make interlaced yarns is at the section $Z=0$ of the air jet nozzle, $R_{d}$ deciding the geometry of the flow region at $Z=0$ has a greater effect on the processability than $L_{d}$.

\section{Conclusions}

In order to make clear the air flow in the yarn duct, which has the maximum effect on the generation of interlaced yarns, both the flow near to the yarn duct wall and the jet issuing from a circular air jet nozzle were measured in basic type interlacers with various diameters and lengths of yarn duct at various supplied air pressures. Results obtained are as follows:

(1) The air in basic type interlacers runs against the duct yarn wall opposite to the air jet nozzle, flows radially along the yarn duct wall, forms two twin spiral eddies, and flows out of the yarn duct edge to the atmosphere. This is no exception even for the diameter ratio $R_{d}=1$.

(2) The flow is biased to the position of $\theta=180^{\circ}$ opposite to the air jet nozzle. As the air approaches the yarn duct edge, the circumferential component of flow velocity becomes small, the axial component becomes dominant and more uniform.

(3) When $R_{d}$ is large, the flow velocity near to the yarn duct wall is small. The flow in the region of small $|Z|$ turns more in the circumferential direction than when $R_{d}$ is small.

(4) When $p$ is large, the flow velocity near to the yarn duct wall is large except near to the air jet nozzle. However, $p$ has no effect on the flow direction near to the yarn duct wall.

(5) When $L_{d}$ is changed, the flow near to the yarn duct wall is not changed at the same non-dimensional axial position $Z$. That is, the flow in the yarn duct depends only on the flow on the upstream side, and is not influenced by the yarn duct edge.

(6) The jet width almost linearly increases with $R_{d}$. It shrinks from the diameter of air jet nozzle when $R_{d}$ is smaller than 1.5, but swells from the exit of air jet nozzle when $R_{d}$ is larger than 1.5. $p$ has no influence on the jet width.

(7) The boundary of core flow could be observed by the Schlieren technique when $R_{d}=2.0$ and 2.4. The width of core flow increases with $p$, although it is smaller than the diameter of air jet nozzle when $p$ is small. Moreover, the detached shock wave was observed in front of the yarn duct 
wall opposite to the air jet nozzle on the experimental condition that $R_{d}=1.0-2.4$ and $p=0.2-0.6 \mathrm{MPa}$.

(8) The processability is more sensitive to $R_{d}$ than to $L_{d}$.

\section{Acknowledgement}

The authors would like to express their gratitude to Professor Fujio Yamamoto, Technician Mr. Ken-ichi Yamauchi and Student Mr. Hiroshi Sakai of University of Fukui.

\section{References}

[1] Rihdahrusutorumu R, Wahto RJ, Panthingu JUW, Neruson TR (1961) Japanese Official Gazette of Patents-Showa 36-12230

[2] Iemoto Y, Chono S, Tanaka T (1990) J Text Mach Soc Japan (predecessor journal of J Text Eng), 43, T10-T17

[3] Iemoto Y, Chono S, Kasamatsu K, Lou W (1992) J Text Mach Soc Japan (predecessor journal of J Text Eng), 45, T1-T9

[4] Iemoto Y, Chono S, Tanida M (1986) J Text Mach Soc Japan (predecessor journal of J Text Eng), 39, T123-T130

[5] Chono S, Iemoto Y, Tanida M (1987) J Text Mach Soc Japan (predecessor journal of J Text Eng), 40, T33-T41

[6] Chono S, Iemoto Y, Han W (1988) J Text Mach Soc Japan (predecessor journal of J Text Eng), 41, T89-T95

[7] Chono S, Iemoto Y, Han W (1989) J Text Mach Soc Japan (predecessor journal of J Text Eng), 42, T106-T113

[8] Chono S, Iemoto Y, Terachi T (1990) J Text Mach Soc Japan (predecessor journal of J Text Eng), 43, T53-T61

[9] Iemoto Y, Chono S, Terachi T (1989) J Text Mach Soc Japan (predecessor journal of J Text Eng), 42, T114-T123

[10] Ge M, Iemoto Y, Tanoue S (1997) J Text Mach Soc Japan (predecessor journal of J Text Eng), 50, T209-T215

[11] Ge M, Iemoto Y, Tanoue S (1998) J Text Mach Soc Japan (predecessor journal of J Text Eng), 51, T175-T181

[12] Ge M, Iemoto Y, Tanoue S (1998) J Text Mach Soc Japan (predecessor journal of J Text Eng), 51, T193-T198

[13] Ge M, Iemoto Y, Tanoue S (1999) J Text Mach Soc Japan (predecessor journal of J Text Eng), 52, T51-T56

[14] Ge M, Iemoto Y, Tanoue S (1999) J Text Mach Soc Japan (predecessor journal of J Text Eng), 52, T122-T129

[15] Lünenschloß J, Zilg J-P (1980) Chemiefasern/Textilindustrie, 30/82, 809-820

[16] Iemoto Y, Chono S (1987) J Text Mach Soc Japan (predecessor journal of J Text Eng), 40, T47-T56

[17] Iemoto Y, Chono S, Qin H, Lou W (1994) J Text Mach Soc Japan (predecessor journal of J Text Eng), 47, T151-T160

[18] Murakami K, Tokunaga K, Nomura S, Naito S, Abe M (2006) J Text Eng, 52, 73-79

[19] Tokunaga K, Murakami K, Kitamura M, Nomura S, Naito S (2006) J Text Eng, 52, 121-129 